\title{
Bose-Einstein condensates in the large-gas-parameter regime
}

\author{
A. Fabrocini ${ }^{1,2}$ and A. Polls ${ }^{3}$ \\ ${ }^{1}$ INFN, Sezione di Pisa, I-56100 Pisa, Italy \\ ${ }^{2}$ Department of Physics E. Fermi, University of Pisa, I-56100 Pisa, Italy \\ ${ }^{3}$ Departament d'Estructura i Constituents de la Matèria, Universitat de Barcelona, E-08028 Barcelona, Spain
}

(Received 30 March 2001; revised manuscript received 19 July 2001; published 16 November 2001)

\begin{abstract}
Bose-Einstein condensates of $10^{4}{ }^{85} \mathrm{Rb}$ atoms in a cylindrical trap are studied using a recently proposed approach based on a local-density approximation. Since the existence of a Feshbach resonance allows for widely tuning the scattering length of the atoms, values of the peak gas parameter $x_{p k}$ of the order of $10^{-2}$ may be attained and the standard analysis based on Gross-Pitaevskii and/or Thomas-Fermi equations may result in being questionable. Energy functionals derived from the correlated basis functions theory and the low-density expansion for a homogeneous hard-spheres gas are used to estimate corrections to the Gross-Pitaevskii equation. The two functionals give similar results, both showing large differences with respect to the Thomas-Fermi and Gross-Pitaevskii ones. The column densities at $z=0$ may differ by as much as $\sim 30 \%$ and the half maximum radius by $\sim 20 \%$. The scattering lengths estimated by fitting the half maximum radius may differ by $\sim 40 \%$ from those given by a Thomas-Fermi-based analysis of the experimental data.
\end{abstract}

DOI: 10.1103/PhysRevA.64.063610

PACS number(s): 03.75.Fi, 05.30.Jp, 32.80.Pj

Bose-Einstein condensation of magnetically trapped alkali-metal atoms has been achieved in several experimental setups, most of them in regimes where the atomic gas is considered to be very dilute, i.e., the average interatomic distance is much larger than the range of the interaction. As a consequence, the physics is dominated by two-body collisions, generally well described in terms of the $s$-wave scattering length $a$. The crucial parameter defining the condition of diluteness is the gas parameter $x(\mathbf{r})=n(\mathbf{r}) a^{3}$, where $n(\mathbf{r})$ is the local density. For low values of the average gas parameter, $x_{a v} \leqslant 10^{-3}$, the mean-field Gross-Pitaevskii (GP) equation [1] is the logical tool to study the system (see Ref. [2] for an extended review).

The gas parameter may be brought outside the regime of validity of the GP equation by two ways: either by increasing the number of atoms $N$ in the condensate, or by changing their effective size. Recent experiments have explored both possibilities. On one side, they have reached very large $N$ values, $N \sim 10^{8}$; on the other, the scattering lengths have been widely tuned. The second approach promises to be much more efficient to reach large $x$ regions.

In a recent experiment performed at (JILA) [3,4], it was possible to confine about $10^{4}$ atoms of ${ }^{85} \mathrm{Rb}$ in a cylindrical trap. By exploiting the presence of a Feshbach resonance at a magnetic field of $B \sim 155$ Gauss, the scattering length was varied from negative to very high-positive values. Actually, it was suggested some time ago [5] that $a$ could be modulated by taking advantage of its expected strong variation in the vicinity of a magnetically induced Feshbach resonance in collisions between cold alkali-metal atoms. Several experiments have supported this proposal by demonstrating this type of scattering length variation for several alkali-metal atoms, as ${ }^{85} \mathrm{Rb}, \mathrm{Cs}$, and $\mathrm{Na}[6-8]$. However, only very recently the Boulder group at JILA has been successful in producing stable ${ }^{85} \mathrm{Rb}$ condensates where $a$ can be effectively tuned over a very wide range.

To explore with confidence such regimes of high values of the parameter $x$, a necessary task is to investigate the accu- racy of the GP equation. Moreover, to quantify the limitations of the GP description is of particular relevance since the empirical estimate of the scattering length is based upon the so called Thomas-Fermi (TF) approximation to the GP equation. The TF approximation amounts to disregard the kinetic energy term in the GP equation. The TF and GP results are expected to coincide for large $N$ and/or $a$ values. In the experimental analysis, a common procedure consists in measuring the column density, given by the integral of the particle density along a direction perpendicular to the symmetry axis of the trap $n_{c}(z)=\int d x n(x, 0, z)$. The $x$ direction coincides with that of the light beam used to image the atomic cloud. Then, the scattering length is inferred by finding the value of $a$ that, in the framework of the TF equation, provides a column density with the same experimental size.

In a previous paper [9], we have proposed a local-density approximation (LDA) based approach, where the correlated basis functions (CBF) [10] theory is used to generate an energy functional able to take into account the correlations induced by the interparticle potential. These correlations are expected to become important in the large $x$ region. The accuracy of the $\mathrm{CBF}$ results for the energy of a homogeneous gas of bosonic hard spheres (HS) was successfully checked up to $x=10^{-3}$ in Ref. [9] against a diffusion Monte Carlo calculation (DMC) [11], providing the exact solution of the many-body Schrödinger equation. We have then checked that the agreement between $\mathrm{CBF}$ and DMC results is still very satisfactory at $x=10^{-2}$. In the same reference, the accuracy of energy functionals derived by truncating at different orders the low-density (LD) expansion of the energy density of the HS homogeneous gas [12] was studied. The two approaches (LDA/CBF and LDA/LD, respectively) provided GP-like equations, whose solution allowed us to estimate the corrections to the GP treatment of hard-sphere bosons in isotropic, harmonic traps.

In this paper, we will solve the LDA/CBF and LDA/LD equations in the case of cylindrical traps, in order to estimate the corrections to the GP and TF results in the conditions of 
the JILA experiment. We begin by briefly recalling the derivation of the LDA equations. Then, in the simpler case of a spherical trap, we will compare different approaches in a situation where the number of atoms and the frequency of the trap are kept fixed and the scattering length is allowed to vary in a representative range of values. The LDA spherical results are also compared with those obtained by solving the modified GP equation of Ref. [13] and a modified version of the TF equation. Finally, we will consider a cylindrical trap corresponding to the experimental setup. Depending on the value of the scattering length, the corrections to the GP results may be as large as $30 \%$ for energy, chemical potential and $\sim 40 \%$ for the extracted scattering length.

For a system of $N$ bosons at $T=0$ temperature, described by the Hamiltonian

$$
H=-\frac{\hbar^{2}}{2 m} \sum_{i} \nabla_{i}^{2}+\sum_{i} V_{e x t}\left(\mathbf{r}_{i}\right)+\sum_{i<j} V\left(\mathbf{r}_{i j}\right)
$$

where $V_{\text {ext }}\left(\mathbf{r}_{i}\right)$ is the external confining potential and $V\left(\mathbf{r}_{i j}\right)$ is the interatomic potential, a CBF ground-state wave function may be written as: $\psi_{C B F}(N)=F(N) \psi_{M F}(N)$, where $F(N)$ is a many-body correlation operator applied to the mean-field wave function (w.f.), $\psi_{M F}(N)$. The advantage of a $\mathrm{CBF}$ w.f. consists mainly in the fact that nonperturbative effects may be easily taken into account by the correlation operator. In particular, this is the case of the short-range repulsion in HS systems.

The CBF energy is given by

$$
\begin{aligned}
E_{C B F}= & \frac{\hbar^{2}}{2 m} \int d \mathbf{r}_{1}\left[\vec{\nabla}_{1} n^{1 / 2}\left(\mathbf{r}_{1}\right)\right]^{2}+\int d \mathbf{r}_{1} n\left(\mathbf{r}_{1}\right) V_{\text {ext }}\left(\mathbf{r}_{1}\right) \\
& +E_{\text {corr }},
\end{aligned}
$$

where the one-body density $n(\mathbf{r})$ is normalized to $N$, and $E_{\text {corr }}$ is the so called correlation energy [9]. In the LDA approach, $E_{c o r r}$ is approximated by $E_{c o r r}^{L D A / C B F}$ defined by

$$
E_{c o r r}^{L D A / C B F}=\int d \mathbf{r}_{1} n\left(\mathbf{r}_{1}\right) e_{H S}^{C B F}\left[n\left(\mathbf{r}_{1}\right)\right] .
$$

$e_{H S}^{C B F}[n(\mathbf{r})]$ is the CBF energy per particle of the HS homogeneous gas at density $n(\mathbf{r})$, and it may be readily computed by the hypernetted chain technique [10]. A minimization of the energy with respect to $n(\mathbf{r})$ is obtained by solving a correlated Hartree-like equation (Eq. (17) of Ref. [9]).

The correlation energy may be estimated in an analogous LDA framework by means of the low-density expansion for the energy of a homogeneous system of hard spheres, whose diameter coincides with the scattering length [12]

$$
\begin{aligned}
e_{H S}^{L D}(n)= & \frac{2 \pi n a \hbar^{2}}{m}\left[1+\frac{128}{15}\left(\frac{n a^{3}}{\pi}\right)^{1 / 2}+8\left(\frac{4}{3} \pi-\sqrt{3}\right)\right. \\
& \left.\times\left(n a^{3}\right) \ln \left(n a^{3}\right)+O\left(n a^{3}\right)\right] .
\end{aligned}
$$

Retaining the first term of the LD expansion to evaluate $E_{c o r r}$ provides the standard GP energy expression, and, con-
TABLE I. Ground-state properties of $N=10^{4}{ }^{85} \mathrm{Rb}$ atoms confined in a spherical trap $(\omega / 2 \pi=12.83 \mathrm{~Hz})$ in different approaches. $\mu_{1}=$ chemical potential, $E_{1} / N=$ energy per atom, $x_{p k}=$ peak gas parameter. Energies in $\mathrm{HO}$ units.

\begin{tabular}{lcccc}
\hline \hline$a / a_{0}$ & 1400 & 3000 & 8000 & 10000 \\
\hline$\mu_{1}^{T F}$ & 13.30 & 18.04 & 26.71 & 29.20 \\
$\mu_{1}^{G P}$ & 13.42 & 18.13 & 26.78 & 29.27 \\
$\mu_{1}^{M G P}$ & 13.97 & 19.84 & 33.38 & 38.06 \\
$\mu_{1}^{C B F}$ & 13.98 & 19.77 & 33.53 & 38.65 \\
$E_{1}^{T F} / N$ & 9.50 & 12.89 & 19.08 & 20.86 \\
$E_{1}^{G P} / N$ & 9.67 & 13.02 & 19.18 & 20.95 \\
$E_{1}^{M G P} / N$ & 10.01 & 14.10 & 23.43 & 26.63 \\
$E_{1}^{C B F} / N$ & 10.03 & 14.06 & 23.38 & 26.76 \\
& & & & \\
$x_{p k}^{T F}$ & $6.27 \times 10^{-4}$ & $3.91 \times 10^{-3}$ & $4.11 \times 10^{-2}$ & $7.02 \times 10^{-2}$ \\
$x_{p k}^{G P}$ & $6.30 \times 10^{-4}$ & $3.91 \times 10^{-3}$ & $4.12 \times 10^{-2}$ & $7.03 \times 10^{-2}$ \\
$x_{p k}^{M G}$ & $5.73 \times 10^{-4}$ & $3.20 \times 10^{-3}$ & $2.60 \times 10^{-2}$ & $4.12 \times 10^{-2}$ \\
$x_{p k}^{C B F}$ & $5.78 \times 10^{-4}$ & $3.25 \times 10^{-3}$ & $2.54 \times 10^{-2}$ & $3.87 \times 10^{-2}$ \\
\hline \hline
\end{tabular}

sequently, the GP equation (after minimizing over the density). By including successive corrections, modified GP (MGP) equations are obtained.

It is worth noticing that, up to these orders of the expansion, the details of the potential do not show up, and any potential with the same scattering length would give identical results. Such an universal behavior was confirmed by the DMC calculation of Ref. [11]. In Refs. [9,11,14] it was shown that the first term of the expansion is accurate only at very low values of $x$ for an uniform system. The addition of the first correction in $x^{1 / 2}$ in the square brackets gives a good representation of the exact DMC results up to $x=10^{-2}$. However, the logarithmic term severely spoils the agreement already at intermediate $x$ values, and it has not been incorporated into the functional energy, $e_{H S}^{L D}(n)$, used in this paper.

As is usually done, it is convenient to simplify the notation by expressing lengths and energies in harmonic oscillator (HO) units. The spatial coordinates, the energy, and the density are rescaled as $\mathbf{r}=a_{H O} \mathbf{r}_{1}, E=\hbar \omega E_{1}$, and $\Psi(\mathbf{r})$ $=n(\mathbf{r})^{1 / 2}=\left(N / a_{H O}^{3}\right)^{1 / 2} \Psi_{1}\left(\mathbf{r}_{1}\right)$, where $\Psi_{1}\left(\mathbf{r}_{1}\right)$ is normalized to unity and $a_{H O}=(\hbar / m \omega)^{1 / 2}$. Using these variables and performing a functional variation of the energy within the LDA/LD scheme truncated at the first order in $x^{1 / 2}$, we obtain the MGP equation for the spherically symmetric trap:

$$
\begin{aligned}
& {\left[-\frac{1}{2} \nabla_{\mathbf{r}_{1}}^{2}+\frac{1}{2} r_{1}^{2}+4 \pi a_{1} N\left|\psi_{1}\left(\mathbf{r}_{1}\right)\right|^{2}\right.} \\
& \left.\quad+\sqrt{\pi a_{1}^{5} N^{3}} \frac{128}{3}\left|\psi_{1}\left(\mathbf{r}_{1}\right)\right|^{3}\right] \psi_{1}\left(\mathbf{r}_{1}\right)=\mu_{1} \psi_{1}\left(\mathbf{r}_{1}\right),
\end{aligned}
$$

where $a_{1}=a / a_{H O}$ and $\mu_{1}$ is the chemical potential in $\mathrm{HO}$ units.

Table I gives some results for $N=10^{4}{ }^{85} \mathrm{Rb}$ atoms confined in a spherical trap with an oscillator angular frequency 
$\omega_{H O} / 2 \pi=\left(\omega_{\perp}^{2} \omega_{z}\right)^{1 / 3} / 2 \pi=12.83 \mathrm{~Hz}$, where $\omega_{\perp} / 2 \pi=17.5$ $\mathrm{Hz}$ and $\omega_{z} / 2 \pi=6.9 \mathrm{~Hz}$ are the radial and axial frequencies associated with the external potential of the cylindrical trap used in Ref. [3]. In the Table, we study the dependence of the energy per particle and of the chemical potential on the scattering length, given in units of the Bohr radius of the Hydrogen atom $a_{0}$. For this trap, $a_{H O}=57523 a_{0}$. We also show the TF results. The main appeal of the TF approximation is that it is possible to derive simple analytical expressions [15] of several quantities. For instance, $\mu_{1}^{\mathrm{TF}}=1 / 2\left(15 a_{1} N\right)^{2 / 5}$. Also reported are the peak values of the gas parameter $x_{p k}=n(0) a^{3}=N a_{1}^{3}\left|\psi_{1}(0)\right|^{2}$, whose $\mathrm{TF}$ estimate is $x_{p k}^{\mathrm{TF}}$ $=\left(15^{2} a_{1}^{12} N^{2}\right)^{1 / 5} /(8 \pi)$.

At low values of the scattering length, the MGP and CBF corrections are small; besides, the TF approach to the GP equation is not fully satisfactory. As expected, the TF and GP results are much closer when $a$ increases, where, however, those corrections become important and of the order of $30 \%$ at the largest value of $a=10000 a_{0}$. As $a$ increases, $x_{p k}$ also increases, the MGP and CBF density distributions get wider and $x_{p k}^{\mathrm{MGP}, \mathrm{CBF}}$ are depleted with respect to both TF and GP because of the repulsive character of the extra interaction terms. The CBF and MGP results are always confortably close to each other. A TF approach to the MGP equation (MTF) was proposed in Ref. [2], by simply dropping the kinetic energy term in Eq. (5). The resulting equation is nonlinear in the density, and looses the property of having analytical solutions, except at the very first step of an iterative procedure [2] (MTF/1). By comparing the MTF/1 approximation with the full solution of the MTF equation, a good agreement at low-scattering lengths is found $\left(\mu_{1}^{\mathrm{MTF}}=13.85\right.$, $x_{p k}^{\mathrm{MTF}}=5.71 \times 10^{-2}, \quad$ and $\quad \mu_{1}^{\mathrm{MTF} / 1}=13.89, \quad x_{p k}^{\mathrm{MTF} / 1}=5.54$ $\times 10^{-2}$ at $a=1400 a_{0}$ ). However, substantial (or even dramatic) differences appear in both the chemical potential and the peak gas parameter at large $a$ values. In fact, at $a$ $=10000 a_{0}$, we obtain $\mu_{1}^{\mathrm{MTF}}=38.00$ and $\mu_{1}^{\mathrm{MTF} / 1}=42.92$, with a $13 \%$ disagreement, whereas $x_{p k}^{\mathrm{MTF}}=4.12 \times 10^{-2}$ and $x_{p k}^{\mathrm{MTF} / 1}$ becomes unrealistically negative.

Our MGP equation has been derived within the LDA, using energy functionals provided by the homogeneous gas of hard spheres. In this way, corrections to the kinetic energy due to the inhomogeneity of the trapped bosons system are not fully and correctly considered. A consistent treatment of these corrections was given in Ref. [13], by taking into account quantum fluctuaction corrections to the mean-field approximation up to $x^{1 / 2}$ terms. This approach gives a MGPlike equation $(\mathrm{MGP} / \mathrm{QF})$ having the term,

$$
\frac{17}{18} \frac{1}{\sqrt{\pi}}\left[2 \sqrt{x} \boldsymbol{\nabla}_{\mathbf{r}_{1}}^{2}+\nabla_{\mathbf{r}_{1}} \sqrt{x} \boldsymbol{\nabla}_{\mathbf{r}_{1}}\right] \psi_{1}\left(\mathbf{r}_{1}\right),
$$

added to the left-hand side (1.h.s) of Eq. (5). We have solved the MGP/QF equation for two of the cases shown in Table I $\left(a / a_{0}=1400\right.$ and 10000) and found marginal differences (less than $0.1 \%$ ) with the results given by the MGP equation, both for the chemical potential and the peak gas parameter.
TABLE II. Ground-state properties of $N=10^{4}{ }^{85} \mathrm{Rb}$ atoms in the cylindrical trap described in the paper. Energies in HO units.

\begin{tabular}{lcccc}
\hline \hline$a / a_{0}$ & 1400 & 3000 & 8000 & 10000 \\
\hline$\mu_{1}^{T F}$ & 9.70 & 13.15 & 19.47 & 21.29 \\
$\mu_{1}^{G P}$ & 9.82 & 13.25 & 19.55 & 21.36 \\
$\mu_{1}^{M G P}$ & 10.22 & 14.51 & 24.38 & 27.79 \\
$\mu_{1}^{C B F}$ & 10.19 & 14.38 & 24.37 & 28.09 \\
$E_{1}^{T F} / N$ & 6.93 & 9.39 & 13.91 & 15.21 \\
$E_{1}^{G P} / N$ & 7.08 & 9.52 & 14.00 & 15.29 \\
$E_{1}^{M G P} / N$ & 7.33 & 10.31 & 17.09 & 19.43 \\
$E_{1}^{C B F} / N$ & 7.31 & 10.23 & 16.98 & 19.42 \\
& & & & \\
$x_{p k}^{T F}$ & $6.23 \times 10^{-4}$ & $3.88 \times 10^{-3}$ & $4.09 \times 10^{-2}$ & $6.98 \times 10^{-2}$ \\
$x_{p k}^{G P}$ & $6.28 \times 10^{-4}$ & $3.90 \times 10^{-3}$ & $4.10 \times 10^{-2}$ & $7.00 \times 10^{-2}$ \\
$x_{p k}^{M G P}$ & $5.72 \times 10^{-4}$ & $3.19 \times 10^{-3}$ & $2.60 \times 10^{-2}$ & $4.10 \times 10^{-2}$ \\
$x_{p k}^{C B F}$ & $5.76 \times 10^{-4}$ & $3.24 \times 10^{-3}$ & $2.53 \times 10^{-2}$ & $3.86 \times 10^{-2}$ \\
\hline \hline
\end{tabular}

The results for the cylindric trap used in the JILA experiments are given in Table II. Most of the TF results are again analytical. The $\mathrm{HO}$ units in the cylindric case are: $\mathbf{r}$ $=a_{\perp, H O} \mathbf{r}_{1}, E=\hbar \omega_{\perp} E_{1}, \Psi(\mathbf{r})=\left(N / a_{\perp, H O}^{3}\right)^{1 / 2} \Psi_{1}\left(\mathbf{r}_{1}\right)$, and $a_{\perp, H O}=\left(\hbar / m \omega_{\perp}\right)^{1 / 2}$. The trap deformation parameter is $\lambda$ $=\omega_{z} / \omega_{\perp}=0.39$.

The column density (already defined in the introductory part of this paper) is an accessible experimental quantity, connected to the density profile. Its TF expression reads

$$
n_{c}^{T F}\left(z_{1}\right)=\frac{2}{12 \pi a_{1} N}\left[2\left(\mu_{1}^{T F}-\frac{1}{2} \lambda^{2} z_{1}^{2}\right)\right]^{3 / 2} .
$$

A measure of the extension of the condensate is the half maximum radius of the column density, $R_{1 / 2}$, defined as the $z_{1}$ value where $n_{c}\left(z_{1}=R_{1 / 2}\right)=(1 / 2) n_{c}(0)$. Also of interest is the full strength at half maximum, FSHM, given by the integrated strength of the column between the $\pm R_{1 / 2}$ values.

In Fig. 1, we show the column densities in different approaches, for the same set of scattering lengths reported in the tables. The solid, dot-dashed and dashed lines correspond to the MGP, CBF, and TF results, respectively; stars are the GP densities; the triangles in the two upper panels give $n_{c}\left(z_{1}\right)$ evaluated in MGP, but changing the scattering length to reproduce $R_{1 / 2, T F}$, supposedly corresponding to the measured radius. The two modified values are $a=5920 a_{0}$ for $R_{1 / 2, T F}=10.20$ and $a=4940 a_{0}$ for $R_{1 / 2, T F}=9.75$ and the related MGP columns are practically identical to the TF ones. The GP and TF results almost coincide and the MGP and $\mathrm{CBF}$ corrections are more sizeable at the two largest values of $a$, where $x_{p k}$ becomes of the order of $10^{-2}$. Because of the repulsive nature of the MGP/CBF extra term, $R_{1 / 2, M G P / C B F}$ are larger than $R_{1 / 2, T F}$, while $\mathrm{FSHM}_{M G P / C B F}$ are smaller than $\operatorname{FSHM}_{T F}$, and for low $z_{1}$ values $n_{c}^{M G P / C B F}\left(z_{1}\right)$ stay below $n_{c}^{T F}\left(z_{1}\right)$. A smaller scattering length is required to reproduce $R_{1 / 2, T F}$ and $\mathrm{FSHM}_{T F}$ in the MGP and CBF approaches in the high $x_{p k}$ region. In fact, we find a reduction of $\sim 40 \%$ of $a / a_{0}$. This analysis shows that using the TF 

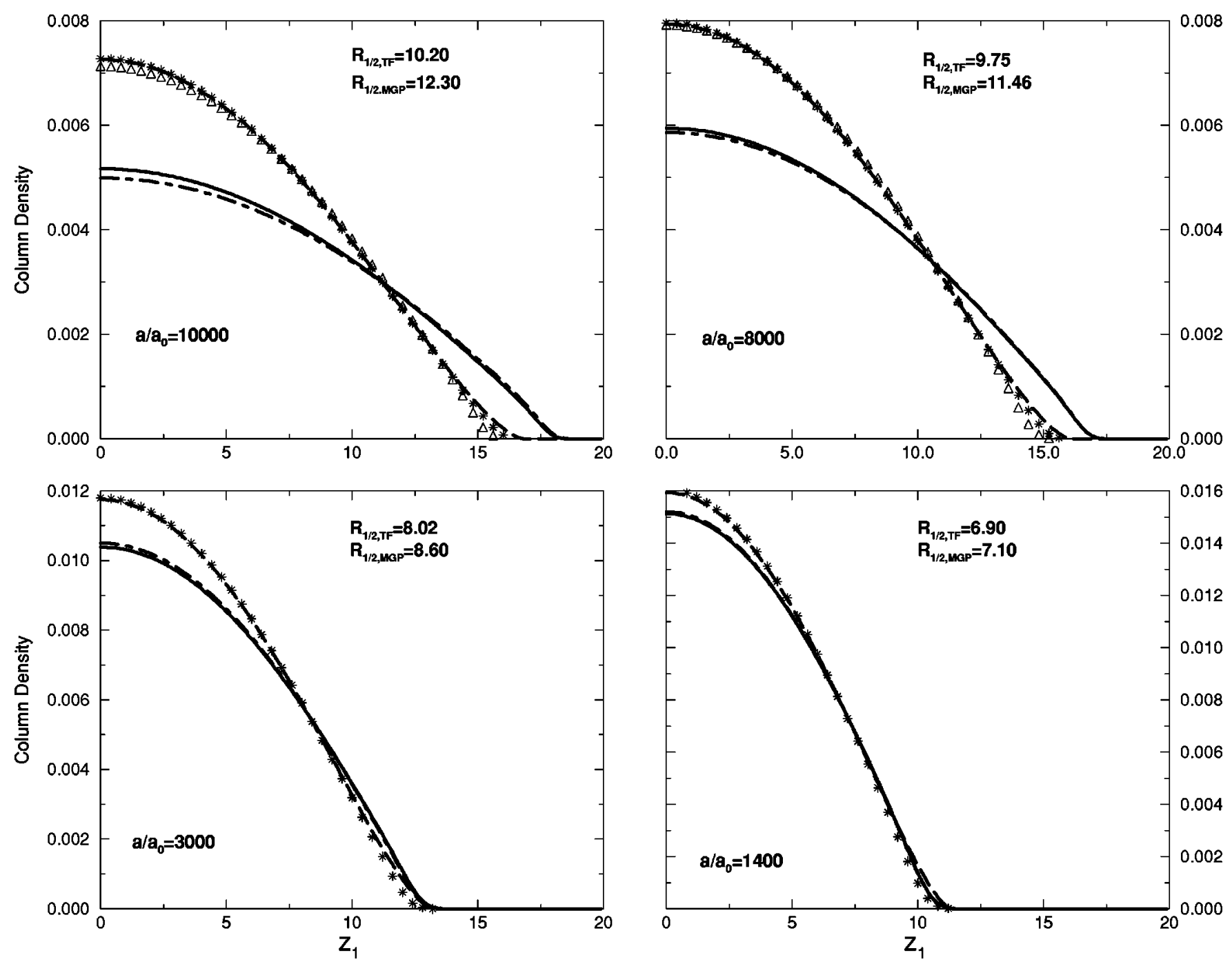

FIG. 1. Column densities at four values of the scattering length for the cylindrical trap. Dashed lines $=$ TF, stars $=\mathrm{GP}$, solid lines $=\mathrm{MGP}$, dot-dashed lines $=\mathrm{CBF}$. The triangles in the first (second) upper panel give the MGP column density at $a / a_{0}=5920(4940)$.
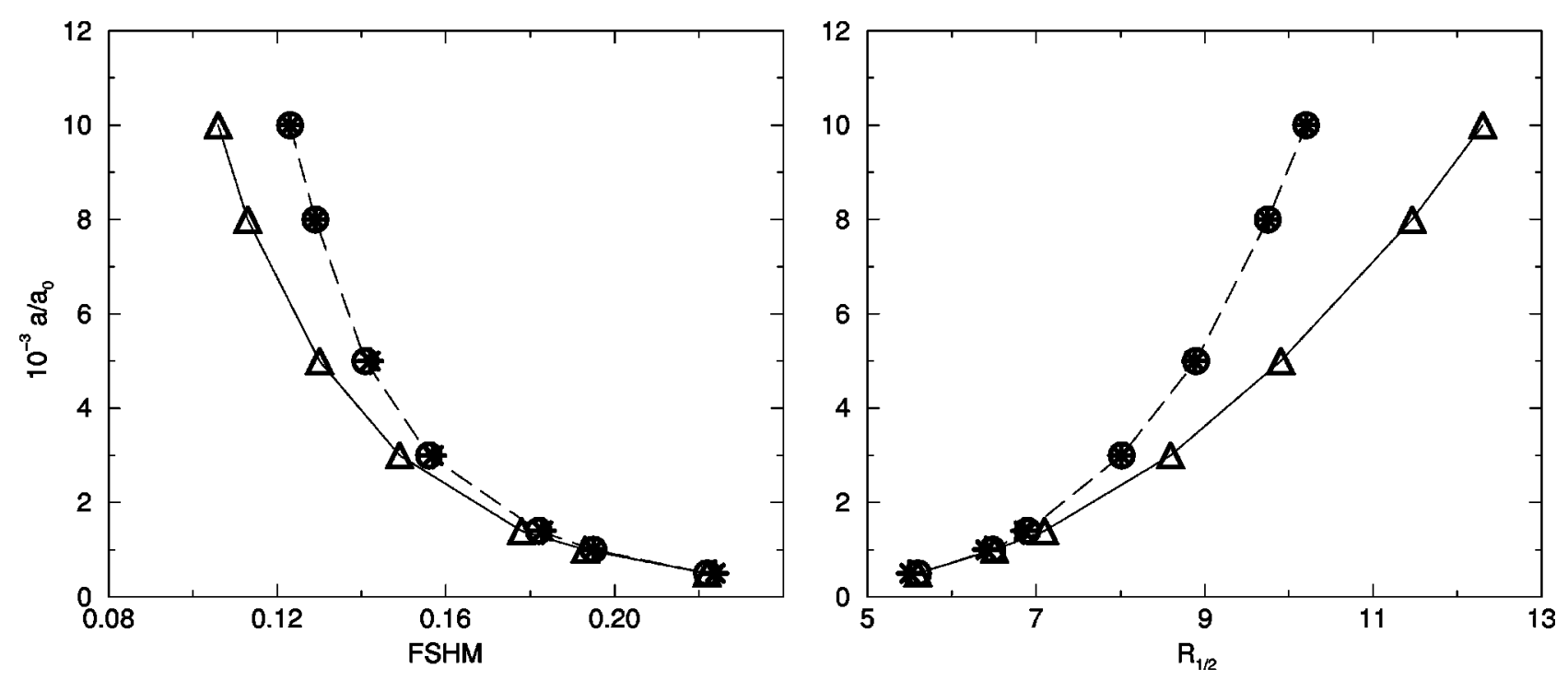

FIG. 2. Scattering length as a function of the full strength at half maximum (left) and of the half maximum radius (right) in the cylindrical trap. Circles, stars, and triangles correspond to the TF, GP, and MGP results, respectively. Lines are a guide to the eyes. 
column density to extract the scattering length in the largegas-parameter regime could lead to severe overestimates in this kind of trap geometry.

The lower panels of the figure roughly correspond to $x_{p k} \sim 10^{-3}-10^{-4}$. As expected, the MGP and CBF corrections are smaller and the computed $R_{1 / 2}$ values become closer when $a / a_{0}$ decreases.

Figure 2 shows the scattering length as a function of FSHM and $R_{1 / 2}$ for the cylindrical trap within the methods we have analyzed. The figure stresses that, depending on the FSHM and $R_{1 / 2}$ values and on the used approach, the estimates of $a$ can differ by up to $40 \%$.

In conclusion, we find that corrections to the TF- and GP-based results may be as large as $30 \%$ in the ground-state properties of the condensate, when the conditions of the JILA experiments for ${ }^{85} \mathrm{Rb}$ are considered. Comparable corrections are obtained for the column densities, where large differences with the standard TF and GP estimates may be found. These differences appear to be relevant for the extraction of the scattering length when large values of the gas parameter come into play. MGP- and the CBF-based approaches are still mean-field theories, since they try to incorporate correlation effects into the average single-particle potential. However, it is conceivable that their predictions are indicative of large corrections due to the interatomic interactions at those regimes attained in recent experiments.

Useful discussions with Ennio Arimondo are gratefully acknowledged. This research was partially supported by DGICYT (Spain) Grant No. PB98-1247, the agreement CICYT (Spain)-INFN (Italy), the program SGR2000-24 from Generalitat de Catalunya, and the Progetto di Ricerca di Interesse Nazionale: Fisica Teorica del Nucleo Atomico e dei Sistemi a Molticorpi from MURST.
[1] L. P. Pitaevskii, Zh. Éksp. Teor. Fiz. 40, 646 (1961) [Sov. Phys. JETP 13, 451 (1961)]; E. P. Gross, Nuovo Cimento 20, 454 (1961).

[2] F. Dalfovo, S. Giorgini, L. Pitaevskii, and S. Stringari, Rev. Mod. Phys. 71, 463 (1999).

[3] S. L. Cornish, N. R. Claussen, J. L. Roberts, E. A. Cornell, and C. E. Wieman, Phys. Rev. Lett. 85, 1795 (2000).

[4] B. Goss, Phys. Today 53, 17 (2000).

[5] W. C. Stwalley, Phys. Rev. Lett. 37, 1628 (1976); E. Tiesinga, B. J. Verhaar, and H. T. C. Stoof, Phys. Rev. A 47, 4114 (1993); E. Tiesinga, A. Moerdijk, B. J. Verhaar, and H. T. C. Stoof, ibid. 46, R1167 (1992).

[6] J. L. Roberts et al., Phys. Rev. Lett. 81, 5109 (1998).

[7] V. Vuletic et al., Phys. Rev. Lett. 82, 1406 (1999).
[8] S. Inouye et al., Nature (London) 392, 151 (1998).

[9] A. Fabrocini and A. Polls, Phys. Rev. A 60, 2319 (1999).

[10] S. Fantoni and A. Fabrocini, in Microscopic Quantum ManyBody Theories and Their Applications, edited by J. Navarro and A. Polls, Lecture Notes in Physics Vol. 510 (SpringerVerlag, Berlin, 1998), p. 119.

[11] S. Giorgini, J. Boronat, and J. Casulleras, Phys. Rev. A 60, 5129 (1999).

[12] A. L. Fetter and J. D. Walecka, Quantum Theory of ManyParticle Sytems (McGraw-Hill, New York, 1971).

[13] E. Braaten and A. Nieto, Phys. Rev. B 56, 14745 (1997).

[14] A. Polls and A. Fabrocini, Condensed Matter Theories (Nova Publishers, New York, in press), Vol. XVI.

[15] M. Edwards and K. Burnett, Phys. Rev. A 51, 1382 (1995). 